\title{
Mejoría clínica de tendinopatía de hombro tras modificaciones ergonómicas del puesto de trabajo de usuarios de ordenador
}

\section{Clinical Improvement of Shoulder Tendinopathy after Ergonomic Modifications of the Workstation of Computer Users}

\author{
Josep María Molina-Aragonés', Narciso Sevilla-Martínez', David Vizcarro-Sanagustín' \\ 1. Centro Corporativo. Barcelona. España.
}

Recibido: 25-06-2019

Aceptado: 16-07-2019

\section{Correspondencia}

Molina Aragonés, Josep Maria

Gran Via de les Corts Catalanes, 587

08007 Barcelona. España.

Tlf: 934824583

josepmariamolina@gencat.cat

Resumen

Introducción: A pesar de la baja evidencia de la relación entre la patología del hombro y el uso de ordenadores, algunos autores asocian dicha patología con el uso del ratón informático, su posición alejada del cuerpo o el espacio insuficiente en la mesa de trabajo.

Caso clínico: Presentamos el caso de cuatro trabajadores que, en el transcurso del último año, se han dirigido espontáneamente al servicio médico manifestando dolor en el hombro que relacionaban con sus condiciones de trabajo y como se ha solucionado dicha clínica mediante la adaptación del puesto de trabajo, básicamente gracias a la introducción de un ratón inalámbrico que ha permitido el rediseño del puesto de trabajo.

Discusión: La imposibilidad de mover el ratón libremente debido a restricciones en el espacio de la mesa de trabajo y a la utilización de ratón más de 4 horas/día durante la jornada laboral se ha descrito como causa de patología a nivel de hombros. En otros casos también se asoció con un espacio insuficiente en la mesa de trabajo, tener el ratón alejado de la posición corporal o utilizarlo durante la mitad o más tiempo de la jornada laboral.

Desde un punto de vista biomecánico el manguito de los rotadores es exigente para evitar fuerzas luxantes, lo que realiza mediante contracción excéntrica o fuerzas neutralizadoras de grupos antagonistas. El supraespinoso tiene un papel importante al inicio de la abducción con un ángulo de tracción máximo aproximadamente a $75^{\circ}$. Determinadas posiciones podrían influenciar su fatiga y/o proceso inflamatorio.

Conclusiones: Nuestra experiencia nos indica que una sencilla y asumible modificación de la posición del hombro durante la utilización del ratón ha sido útil para revertir cuadros clínicamente compatibles con tendinopatías a este nivel.

Med Segur Trab (Internet). 2019;65(256):233-237

Palabras clave (DeCS): Interfaz usuario-ordenador. Periféricos de ordenador. Tendinopatía. Hombro

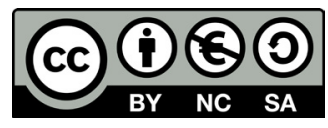

https://creativecommons.org/licenses/by-nc-sa/4.0/ 


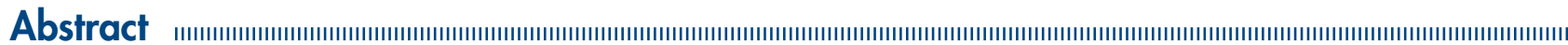

Introduction: Despite the low evidence of the relationship between shoulder pathology and the use of computers, some authors associate this pathology with the use of the computer mouse, its position away from the body or insufficient space on the desk.

Clinical case: We present the case of four workers who, during the last year, consulted medical services spontaneously, manifesting shoulder pain related to their working conditions and how this clinic has been solved by adapting the workplace, basically thanks to the introduction of a wireless mouse that allowed the redesign of the workplace.

Discussion: The inability to move the mouse freely due to restrictions in the space of the work table and the use of mice more than 4 hours / day during the workday has been described as a cause of shoulder pathology. In other cases, it was also associated with insufficient space on the work table, having the mouse away from body position or using it during half or more of the workday.

From a biomechanical point of view the rotator cuff is demanding to avoid dislocating forces, which it does by means of eccentric contraction or neutralizing forces of antagonist groups. The supraspinatus plays an important role at the beginning of abduction with a maximum traction angle of approximately $75^{\circ}$. Certain positions may influence your fatigue and / or inflammatory process.

Conclusions: Our experience indicates that a simple and acceptable modification of the position of the shoulder during the use of the mouse has been useful to improve events of clinical presentations like tendinopathies at this level.

Med Segur Trab (Internet). 2019;65(256):233-237

Keywords (DeCS): User-Computer Interface. Computer peripherals. Tendinopathy. Shoulder 
La investigación de las enfermedades ha avanzado tanto que cada vez es más difícil encontrar a alguien que esté completamente sano. Aldous Huxley.

\section{INTRODUCCIÓN}

La evidencia de la relación entre la patología del hombro y el uso de equipos informáticos es, en general, baja y/o contradictoria ${ }^{1,2,3}$, si bien algunos autores ${ }^{4,5}$ lo asocian con el uso del ratón informático, su posición alejada del cuerpo o el espacio insuficiente en la mesa de trabajo.

\section{Caso clínico}

Presentamos el caso de cuatro trabajadores que, en el transcurso del último año, se han dirigido espontáneamente al servicio médico manifestando dolor en el hombro que relacionaban con sus condiciones de trabajo.

Nuestro objetivo es exponer como una serie de casos de dolor en el hombro en trabajadores usuarios de ordenador se han solucionado clínicamente gracias a la colaboración entre el personal técnico y sanitario del servicio de prevención, mediante la adaptación del puesto de trabajo, básicamente gracias a la introducción de un ratón inalámbrico.

En todos los casos destacaba clínicamente la presencia de positividad de la maniobra de Jobe, sin acompañarse de limitaciones funcionales evidentes. La anamnesis no indicaba motivos de sospecha de riesgo extralaboral. La evaluación ergonómica del puesto de trabajo mostró la existencia de un ratón desplazado a la parte lateral de la superficie de la mesa de trabajo, con restricción de movimientos debido a la disposición del cableado, situación que obligaba al hombro a adoptar una posición permanente de rotación externa y discreta abducción, con una angulación cercana a los $45^{\circ}$ (Figura 1).

Se propuso la utilización de un ratón inalámbrico dispuesto en la zona anterior del cuerpo, trasladando el hombro a rotación interna, al mismo tiempo que se informó sobre las condiciones de uso en esta posición. Se actuó sobre las dimensiones del mobiliario (silla y mesa de trabajo) para disminuir la angulación del hombro (Figura 2). Desde el punto de vista estrictamente médico se prescribió un tratamiento antiinflamatorio al uso por espacio de 5 a 7 días.

\section{Figura 1}

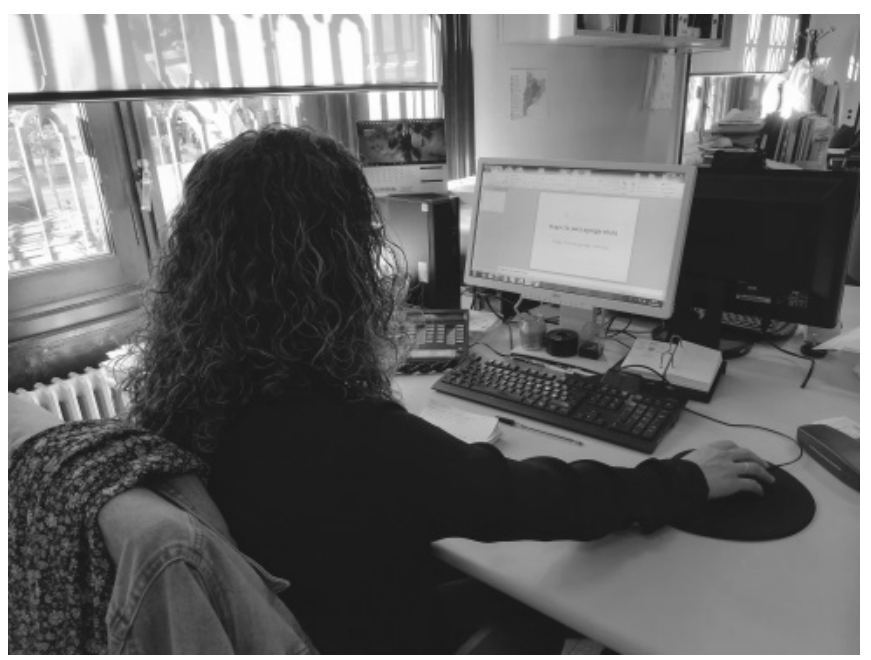

El control evolutivo de estos trabajadores ha permitido constatar la desaparición de la clínica álgica y, hasta la fecha, no han desarrollado nuevos episodios de características similares. 
Figura 2

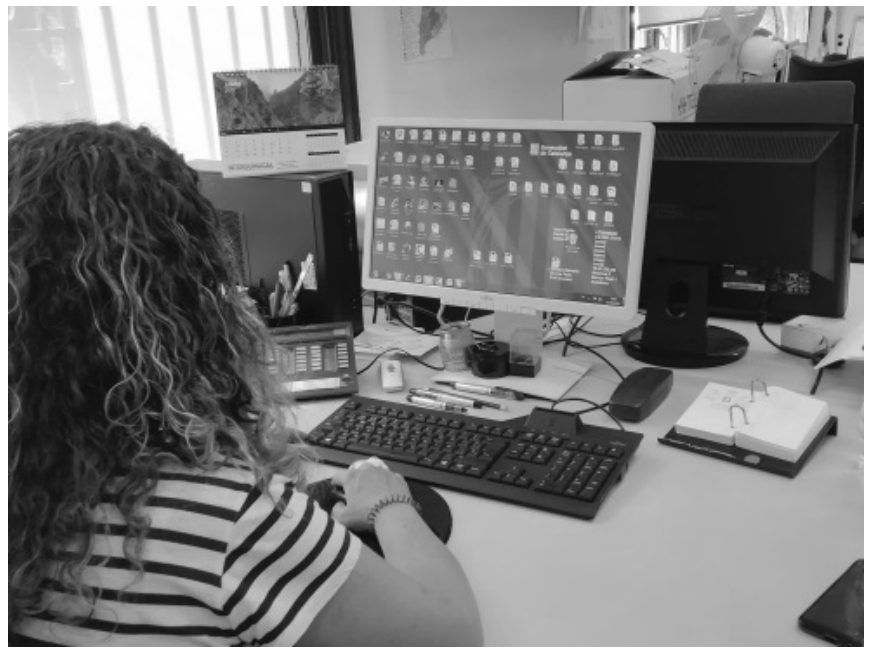

\section{DISCUSIÓN}

Hace unos meses publicamos en esta misma revista una revisión sistemática ${ }^{1}$ sobre las alteraciones asociadas al uso de puestos de trabajo informáticos: en el caso de los hombros, concluíamos que la evidencia de la sintomatología a este nivel y el uso de los equipos era contradictoria. En esta misma línea se manifestaron Wærsted et $\mathrm{al}^{2}$. quienes concluyeron que existía evidencia insuficiente entre las tendinopatias de hombro y su relación con el uso de teclados o ratones. Hoe et al. ${ }^{3}$ también concluyen que existe bajamoderada evidencia de que el apoyo de los brazos o el diseño alternativo de ratones informáticos pueda disminuir significativamente los problemas músculo-esqueléticos de hombros y región cervical en trabajadores de oficina.

Sin embargo, en nuestra revisión -y acorde con la evolución de nuestra serie-, un estudio de elevada calidad metodológica relacionaba la patología de hombros con la adopción de determinadas posturas. Según Huysmans et al. ${ }^{4}$, el riesgo incrementado de alteraciones en extremidades superiores se asociaba a la imposibilidad de mover el ratón libremente debido a restricciones en el espacio de la mesa de trabajo y a la utilización de ratón más de 4 horas/día durante la jornada laboral, del mismo modo que Kiss et al..$^{5}$ en un estudio multicéntrico sobre patología de extremidades superiores en usuarios de PVD, llegaron a la conclusión que los factores relacionados con el uso del ratón informático eran el espacio insuficiente en la mesa de trabajo, tener el ratón alejado de la posición corporal o utilizarlo durante la mitad o más tiempo de la jornada laboral.

Desde un punto de vista biomecánico, y en referencia al manguito de los rotadores del hombro, dada la escasez de estabilidad ósea en esta articulación, la fuerza generada por un músculo requiere siempre de la activación de un músculo antagonista de modo que no se produzca una fuerza luxante, tarea que realiza mediante la contracción excéntrica o mediante la producción de una fuerza neutralizadora en sentido opuesto ${ }^{6}$. El rotador externo primario del húmero es el $\mathrm{m}$. infraespinoso; el $\mathrm{m}$. subescapular es igualmente activo pero tiene un papel antagonista como principal estabilizador del desplazamiento anterior de la cabeza humeral en rotación externa ${ }^{6}$. Estudios electromiográficos también han mostrado que supraespinoso y deltoides se activan a lo largo del rango de elevación del brazo. El supraespinoso, sin embargo, tiene un papel más importante al inicio de la abducción. Su ángulo de tracción es más constante aproximadamente a $75^{\circ}$ y el resto de músculos del manguito ejercen presión en un ángulo cercano a los $45^{\circ}$, provocando fuerzas que estabilizan la articulación glenohumeral $^{6}$. 


\section{CONCLUSIONES}

Si bien la evidencia sigue siendo poca e insuficiente, algunos autores ${ }^{2,3}$ relacionan el uso del ratón de ordenador con tendinopatías del manguito de los rotadores. Nuestra experiencia en estos casos nos indica que una sencilla y asumible modificación de la posición del hombro durante la utilización del ratón ha sido útil para revertir cuadros clínicamente compatibles con tendinopatías a este nivel.

Alentamos a otros compañeros a comunicar experiencias similares o a implicarse en el diseño de estudios que contemplen la hipótesis de la relación de una postura mantenida en rotación externa por el uso o posición indebida del ratón informático con la aparición de cuadros de tendinopatía del manguito de los rotadores.

\section{REFERENCIAS BIBLIOGRÁFICAS}

1. Molina-Aragonés JM, Forns-Carbonell J, Rodriguez-Moreno JM, Sol-Vidiella JM, López-Pérez C. Revisión sistemática sobre las alteraciones óculo-visuales y músculo-esqueléticas asociadas al trabajo con pantallas de visualización de datos. Med Segur Trab (Internet). 2017;63(247):167-205.

2. Wærsted M, Hanvold TN, Veiersted KB. Computer work and musculoskeletal disorders of the neck and upper extremity: A systematic review. BMC Musculoskelet Disord. 2010; 11: 79. doi: 10.1186/1471-2474-11-79

3. Hoe VCW, Urquhart DM, Kelsall HL, Zamri EN, SimMR. Ergonomic interventions for preventing workrelated musculoskeletal disorders of the upper limb and neck among office workers (Review). Cochrane Database of Systematic Reviews 2018, Issue 10. Art. No.: CD008570.

4. Huysmans MA. IJmker S. Blatter BM. Knol DL. Van Mechelen W. Bongers PM. Van der Beek AJ. The relative contribution of work exposure, leisure time exposure and individual characteristics in the onset of arm-wrist-hand and neck-shoulder symptoms among office workers. Int Arch Occup Environ Health. 2012; 85:651-666.

5. Kiss P, De Meester M, Kruse A, Chavée B, Braeckman L. Neck and shoulder complaints in computer workers and associated easy to assess occupational factors-a large-scale cross-sectional multivariate study Int Arch Occup Environ Health. 2012 Feb;85(2):197-206.

6. Nordin M. Frankel VH. Biomecánica bàsica del sistema musculo-esquelético. Capítulo 12 Biomecánica del hombro. Della Valle CJ. Rokito AS. Birdzell MG. Zuckerman JD. Mc Graw Hill-Ineramericana. Madrid. 2004. 